Cornelius Greither · Radan Kučera

\title{
Washington units, semispecial units, and annihilation of class groups
}

Received: 28 November 2019 / Accepted: 19 August 2020 /

Published online: 7 September 2020

\begin{abstract}
Special units are a sort of predecessor of Euler systems, and they are mainly used to obtain annihilators for class groups. So one is interested in finding as many special units as possible (actually we use a technical generalization called "semispecial"). In this paper we show that in any abelian field having a real genus field in the narrow sense all Washington units are semispecial, and that a slightly weaker statement holds true for all abelian fields. The group of Washington units is very often larger than Sinnott's group of cyclotomic units. In a companion paper we will show that in concrete families of abelian fields the group of Washington units is much larger than that of Sinnott units, by giving lower bounds on the index. Combining this with the present paper gives strong annihilation results.
\end{abstract}

By an abelian field, we will always mean a finite abelian extension $K$ of $\mathbb{Q}$. The first aim of this note is to show that under a suitable definition of semispecialness all Washington units of an abelian field with real genus field (in the narrow sense) are semispecial. Washington units are a certain kind of circular units; for the precise definition see below. This occupies the first section. In the second section, we prove a somewhat weaker result for abelian fields whose genus field is imaginary; this will not be used in the sequel.

The main reason why one is interested in semispecial numbers or units in an abelian field $K$ is that they lead to annihilation statements for the class group of $K$. Semispecial units are a generalization of Rubin's notion of "special units" (see [7]), and these special units were used by Rubin, based on pioneering work of Thaine, to obtain annihilation statements for class groups. Shortly after that, the theory of Euler systems was developed, which has led to spectacular results about the structure of class groups and Main Conjectures, but we will not be concerned with Euler systems here. It has turned out that in some situations, special units are hard to come by, so we were led to the notion of "semispecial units", which is maybe less pleasant to the eye but more pliable, and still can be used to obtain annihilation results for class groups. In the last section of this note, we will explain why an annihilation result of this kind that was proved in earlier work of the authors

C. Greither ( $\varangle$ ): UniBw München, 85577 Neubiberg, Germany.

e-mail: cornelius.greither@unibw.de

R. Kučera: Masaryk University, 61137 Brno, Czech Republic.

e-mail: kucera@math.muni.cz

Mathematics Subject Classification: 11R20

https://doi.org/10.1007/s00229-020-01241-y 
for a very restricted class of real abelian fields remains valid for many more abelian fields. The immediate purpose of this is to lay the ground for another paper of ours, in which we treat a particular class of abelian fields $K$ with $\ell$-elementary Galois group, where $\ell$ is an odd prime. For more indications what we can prove about them and why we consider this class to be interesting, see the third section. For the moment, let us just say that the fields in that class have interesting Washington units, obtainable as deep roots of Sinnott units, and that the annihilation result made available in the present paper does produce nontrivial and new insight on the structure of the class group of fields in the subclass, and that the class number of these fields is highly $\ell$-divisible.

We close this introduction by defining semispecial elements (so the reader will know what the next sections are all about), and recalling the notion of Washington units. Let us fix an odd prime number $\ell$. The ring of integers in any number field $L$ will be written $\mathcal{O}_{L}$; conforming with this, the group of units attached to $L$ is written $\mathcal{O}_{L}^{\times}$

Definitions. (i) Let $K$ be any abelian field and let $M$ be any power of $\ell$. For any prime $q \equiv 1(\bmod M)$ let $K(q)$ be the compositum of $K$ with the cyclic field $\mathbb{Q}(q)$ of absolute degree $M$ and conductor $q$. Let $P$ be the set of rational primes that ramify in $K$.

(ii) Let $\mathcal{Q}_{M}$ denote the set of all prime numbers $q$ that are totally split in $K$, congruent to $1+M$ modulo $M^{2}$ and have the property that each $p \in P$ is an $M$ th power modulo $q$.

(iii) An element $\varepsilon \in K^{\times}$will be called $M$-semispecial if for all but finitely many $q$ in $\mathcal{Q}_{M}$, there exists $\varepsilon_{q} \in \mathcal{O}_{K(q)}^{\times}$satisfying two conditions, called "norm condition" and "congruence condition" respectively:

- $\mathrm{N}_{K(q) / K}\left(\varepsilon_{q}\right)=1$;

- If $\tilde{q}$ is the product of all primes of $K(q)$ dividing $q$, then $\varepsilon$ and $\varepsilon_{q}$ have the same image in $\left(\mathcal{O}_{K(q)} / \tilde{q}\right)^{\times} /\left(\left(\mathcal{O}_{K(q)} / \tilde{q}\right)^{\times}\right)^{M}$.

(iv) An element $\varepsilon \in K^{\times}$will be called semispecial (with respect to the fixed prime $\ell$ ) if for any $\ell$-power $M^{\prime}$ there exists a larger $\ell$-power $M$ such that $\varepsilon$ is $M$ semispecial.

The group of cyclotomic units is, in principle, only defined if $K$ is a full cyclotomic field. For any abelian field $K$, one has the group of Sinnott circular units. This group is, roughly speaking, generated by norms of cyclotomic units from suitably chosen cyclotomic fields. Every Sinnott unit is semispecial; this is a fairly easy consequence of the distribution relations for cyclotomic units. If $K$ is an abelian field, an element $x \in K$ is called a Washington unit if it is a cyclotomic unit in the smallest cyclotomic field containing $K$. In particular, if $K$ is itself a full cyclotomic field, then Washington units, Sinnott units, and cyclotomic units are all the same thing. In general, the group of Washington units contains the group of Sinnott units, and we are particularly interested in cases where this inclusion is proper, in a way that can be quantified and exploited. 


\section{The case where the genus field is real}

Let $K$ be an abelian field whose genus field (in the narrow sense) is real. In particular $K$ itself is totally real. We intend to prove that every Washington unit of $K$ is semispecial with respect to any odd prime number $\ell$.

Let $p_{1}, \ldots, p_{s}$ be the primes ramified in $K / \mathbb{Q}$. For each $j=1, \ldots, s$ let $K_{j}$ be the abelian field ramified only at $p_{j}$ such that $\bar{K}=\prod_{j=1}^{s} K_{j}$ is the genus field in the narrow sense of $K$, let $p_{j}^{a_{j}}$ be the conductor of $K_{j}$. As said just above, in this section we assume $\bar{K}$ to be real.

Let us fix an integer $M>1$ (usually large) and a prime $q \equiv 1(\bmod 2 M)$ such that $q$ splits completely in $K / \mathbb{Q}$ and, for each $j=1, \ldots, s$, the prime $p_{j}$ is an $M$ th power modulo $q$. Let $\mathbb{Q}(q)$ be the subfield of the $q$ th cyclotomic field of absolute degree $M$ and for any abelian field $F$ let $F(q)$ be the compositum of $F$ and $\mathbb{Q}(q)$. Notice that $\bar{K}(q)$ is real.

Let $G=\operatorname{Gal}(\bar{K}(q) / \mathbb{Q}(q))$ and $H=\operatorname{Gal}(\bar{K}(q) / K(q))$. The restriction gives isomorphisms $G \cong \operatorname{Gal}(\bar{K} / \mathbb{Q})$ and $H \cong \operatorname{Gal}(\bar{K} / K)$; we shall identify these groups via these isomorphisms. We shall also need $G_{(q)}=\operatorname{Gal}(\bar{K}(q) / \mathbb{Q})$.

To make the notation easier, let $p_{s+1}=q, a_{s+1}=1$ and $K_{s+1}=\mathbb{Q}(q)$. For any nonempty $J \subseteq\{1, \ldots, s+1\}$ we introduce $n_{J}=\prod_{j \in J} p_{j}^{a_{j}}, \zeta_{J}=\exp \left(\frac{2 \pi i}{n_{J}}\right)$, $K_{J}=\prod_{j \in J} K_{j}$, and

$$
\eta_{J}=\mathrm{N}_{\mathbb{Q}\left(\zeta_{J}\right) / K_{J}}\left(1-\zeta_{J}\right) .
$$

We introduce the group of totally positive circular numbers of $\bar{K}$ and $\bar{K}(q)$ as the following Galois modules

$$
\begin{aligned}
\mathcal{D}^{+} & =\left\langle\eta_{J} \mid \emptyset \neq J \subseteq\{1, \ldots, s\}\right\rangle_{\mathbb{Z}[G]} \subset \bar{K}^{\times}, \\
\mathcal{D}_{(q)}^{+} & =\left\langle\eta_{J} \mid \emptyset \neq J \subseteq\{1, \ldots, s+1\}\right\rangle_{\mathbb{Z}\left[G_{(q)}\right]} \subset \bar{K}(q)^{\times} .
\end{aligned}
$$

Theorem 1. There is a homomorphism of $\mathbb{Z}[G]$-modules $\Psi: \mathcal{D}^{+} \rightarrow \mathcal{D}_{(q)}^{+}$satisfying $\Psi\left(\eta_{J}\right)=\eta_{J \cup\{s+1\}}$ for any nonempty $J \subseteq\{1, \ldots, s\}$.

Proof. Denote $I=\{1, \ldots, s\}$. Let $U=\left\langle\rho_{J} ; J \subseteq I\right\rangle_{\mathbb{Z}[G]}$ be the modified Sinnott module defined in [2] with $v=s$, for $T_{i}=\operatorname{Gal}\left(\bar{K} / K_{I-\{i\}}\right)$ being the inertia group of $p_{i}$ in $G$ and $\lambda_{i} \in G$ being the Frobenius automorphism of $p_{i}$ on $\bar{K}$ with trivial action on $K_{i}$ (Frobenius is determined modulo $T_{i}$ only). The circular numbers are well-known to satisfy the following norm relations

$$
\prod_{\tau \in T_{i}} \eta_{J}^{\tau}=\mathrm{N}_{K_{J} / K_{J-\{i\}}}\left(\eta_{J}\right)=\eta_{J-\{i\}}^{1-\lambda_{i}^{-1}} \quad \text { for each } J \subseteq I \text {, and each }\{i\} \subsetneq J,
$$

and

$$
\prod_{\tau \in T_{j}} \eta_{\{j\}}^{\tau}=\mathrm{N}_{K_{j} / \mathbb{Q}}\left(\eta_{\{j\}}\right)=p_{j} \quad \text { for each } j \in I .
$$

The presentation of $U$ is given in [2, Corollary 1.6(ii)]. However we have to take this opportunity to correct the following misprints appearing there. Instead of the 
sharp inclusion $N \subsetneq I$, there should simply be the inclusion $N \subseteq I$ in both (1.7) and (1.9) of loc.cit. Notice that the generator $\rho_{I}=s(G)$ does not interact with the other generators in (1.9), meaning that no relation involves both that generator and any of the others, and that it does not appear in (1.10) loc.cit. at all. Therefore we have a direct sum decomposition $U=\left\langle\rho_{J} ; J \subsetneq I\right\rangle_{\mathbb{Z}[G]} \oplus(s(G) \mathbb{Z})$, and hence

$$
\phi\left(\rho_{J}\right)=\eta_{I-J} \quad \text { for each } J \subsetneq I, \quad \phi\left(\rho_{I}\right)=1,
$$

defines a surjective homomorphism $\phi: U \rightarrow \mathcal{D}^{+}$of $\mathbb{Z}[G]$-modules. Since the $\mathbb{Z}$-rank of $U$ is $[\bar{K}: \mathbb{Q}]+s$ and the $\mathbb{Z}$-rank of $\mathcal{D}^{+}$is $[\bar{K}: \mathbb{Q}]+s-1$, and $U$ has no $\mathbb{Z}$-torsion, we obtain $\operatorname{ker} \phi=\rho_{I} \mathbb{Z}=s(G) \mathbb{Z}$. This implies the existence of an injective homomorphism of $\mathbb{Z}[G]$-modules $\psi: \mathcal{D}^{+} \rightarrow U$ such that

$$
\psi\left(\eta_{I-J}\right)=\rho_{J} \quad \text { for each } J \subsetneq I .
$$

Since the prime $p_{i}$ is an $M$ th power modulo $q$, the Frobenius automorphism of $p_{i}$ on $\bar{K}(q)$ with trivial action on $K_{i}$ is equal to $\lambda_{i}$ for each $i \in I$. Hence for each $J \subseteq I$ and each $i \in J$ we have

$$
\prod_{\tau \in T_{i}} \eta_{J \cup\{s+1\}}^{\tau}=\eta_{J \cup\{s+1\}-\{i\}}^{1-\lambda_{i}^{-1}}
$$

and $[2$, Corollary 1.6(ii)] implies that

$$
\tilde{\phi}\left(\rho_{J}\right)=\eta_{I \cup\{s+1\}-J} \quad \text { for each } J \subsetneq I, \quad \tilde{\phi}\left(\rho_{I}\right)=1,
$$

defines a homomorphism $\tilde{\phi}: U \rightarrow \mathcal{D}_{(q)}^{+}$of $\mathbb{Z}[G]$-modules. Now it is clear that $\Psi=\tilde{\phi} \circ \psi$ satisfies the desired properties.

Theorem 2. Let $\operatorname{Frob}(q)$ be the Frobenius automorphism of $q$ on $\bar{K}$. For any $\varepsilon \in \mathcal{D}^{+}$ we have

$$
\begin{aligned}
\varepsilon^{(q-1) / M} & \equiv \Psi\left(\varepsilon^{\operatorname{Frob}(q)}\right) \text { modulo each prime ideal of } \bar{K}(q) \text { above } q, \\
\mathrm{~N}_{\bar{K}(q) / \bar{K}}(\Psi(\varepsilon)) & =\varepsilon^{1-\operatorname{Frob}(q)^{-1}} .
\end{aligned}
$$

Proof. Since all numbers in $\mathcal{D}^{+}$are relatively prime to $q$, it is enough to prove the statement for generators $\eta_{J}, \emptyset \neq J \subseteq\{1, \ldots, s\}$, of $\mathcal{D}^{+}$. We have

$$
\mathrm{N}_{\bar{K}(q) / \bar{K}}\left(\Psi\left(\eta_{J}\right)\right)=\mathrm{N}_{\bar{K}(q) / \bar{K}}\left(\eta_{J \cup\{s+1\}}\right)=\eta_{J}^{1-\operatorname{Frob}(q)^{-1}} .
$$

There are $a, b \in \mathbb{Z}$ such that $a n_{J}+b q=1$, hence, modulo each prime ideal of $\mathbb{Q}\left(\zeta_{J \cup\{s+1\}}\right)$ above $q$,

$$
\zeta_{J \cup\{s+1\}}=\exp \left(\frac{2 \pi i}{q n_{J}}\right)=\zeta_{\{s+1\}}^{a} \cdot \zeta_{J}^{b} \equiv \zeta_{J}^{b},
$$

and so

$$
1-\zeta_{J \cup\{s+1\}} \equiv 1-\zeta_{J}^{b}=\left(1-\zeta_{J}\right)^{\operatorname{Frob}(q)^{-1}}
$$


Taking norms with respect to $\mathbb{Q}\left(\zeta_{J \cup\{s+1\}}\right) / \mathbb{Q}\left(\zeta_{J}\right)(q)$ we get

$$
\mathrm{N}_{\mathbb{Q}\left(\zeta_{J} \cup\{s+1\}\right) / \mathbb{Q}\left(\zeta_{J}\right)(q)}\left(1-\zeta_{J \cup\{s+1\}}\right) \equiv\left(\left(1-\zeta_{J}\right)^{\operatorname{Frob}(q)^{-1}}\right)^{(q-1) / M},
$$

and taking norms with respect to $\mathbb{Q}\left(\zeta_{J}\right)(q) / K_{J \cup\{s+1\}}$ we finally obtain

$$
\begin{aligned}
\Psi\left(\eta_{J}\right)=\eta_{J \cup\{s+1\}} & =\mathrm{N}_{\mathbb{Q}\left(\zeta_{J \cup\{s+1\}}\right) / K_{J \cup\{s+1\}}\left(1-\zeta_{J \cup\{s+1\}}\right)} \\
& \equiv \mathrm{N}_{\mathbb{Q}\left(\zeta_{J}\right) / K_{J}}\left(\left(1-\zeta_{J}\right)^{\operatorname{Frob}(q)^{-1}}\right)^{(q-1) / M}=\left(\eta_{J}^{\operatorname{Frob}(q)^{-1}}\right)^{(q-1) / M} .
\end{aligned}
$$

In the last-but-one step (the $\equiv$ sign) we have used the congruence (1) above, and two more things: the fields $K_{J}(q)$ and $K_{J \cup\{s+1\}}$ are the same by definition, and since $1-\zeta_{J}$ is in $\mathbb{Q}\left(\zeta_{J}\right)$, it makes no difference whether we apply $\mathrm{N}_{\mathbb{Q}\left(\zeta_{J}\right)(q) / K_{J}(q)}$ or $\mathrm{N}_{\mathbb{Q}\left(\zeta_{J}\right) / K_{J}}$.

The theorem follows.

We now are able to prove that $\varepsilon \in \mathcal{D}^{+} \cap K$ is always semispecial. This is already almost contained in the following result.

Corollary 3. For any $\varepsilon \in \mathcal{D}^{+} \cap K$ we have $\Psi(\varepsilon) \in K(q)$,

$$
\varepsilon^{(q-1) / M} \equiv \Psi(\varepsilon) \text { modulo each prime ideal of } \bar{K}(q) \text { above } q,
$$

and $\mathrm{N}_{\bar{K}(q) / \bar{K}}(\Psi(\varepsilon))=1$.

Proof. For each $\tau \in H$ we have $\varepsilon^{1-\tau}=1$ and so $\Psi(\varepsilon)^{1-\tau}=1$. The rest follows from the assumption that $q$ splits completely in $K / \mathbb{Q}$.

Recall that we are assuming: $K$ is an abelian field whose genus field in the narrow sense is real. We now can show:

Proposition 4. Every $\varepsilon \in \mathcal{D}^{+} \cap K$ is $M$-semispecial for any $\ell$-power $M$, and in particular semispecial (with respect to any odd prime $\ell$ ). This applies in particular if $\varepsilon$ is a Washington unit in $K$.

Proof. From the hypothesis $q \equiv 1+M\left(\bmod M^{2}\right)$ we see that $(q-1) / M \equiv 1$ $(\bmod M)$. This, taken together with Corollary 3 and the fact that by Theorem 1 the image of $\Psi$ consists of units only, gives the statement at once.

\section{The case when the genus field is imaginary}

In this section we drop the assumption of the previous section that the field $K$ and its genus field in the narrow sense are real. That is, we simply assume that $K$ is an abelian field. In this situation we will only obtain a somewhat weaker result, and therefore this section is interesting only for fields whose genus field is imaginary. Moreover this weaker result will not be used in the rest of this paper.

Again we assume that we are given an integer $M$ and a prime $q$ fulfilling the same assumptions as in the previous section. Instead of working in $\bar{K}$ and $\bar{K}(q)$, 
in this section we shall work in $\mathbb{Q}\left(\zeta_{I}\right)$ and $\mathbb{Q}\left(\zeta_{I}\right)(q)$, where $I=\{1, \ldots, s\}$. Hence in this section we define

$$
\begin{aligned}
G & =\operatorname{Gal}\left(\mathbb{Q}\left(\zeta_{I}\right)(q) / \mathbb{Q}(q)\right), \\
H & =\operatorname{Gal}\left(\mathbb{Q}\left(\zeta_{I}\right)(q) / K(q)\right) .
\end{aligned}
$$

For each $i \in I$ we enlarge the inertia subgroup

$$
G_{i}=\operatorname{Gal}\left(\mathbb{Q}\left(\zeta_{I}\right)(q) / \mathbb{Q}\left(\zeta_{I-\{i\}}\right)(q)\right)
$$

by a new element $g_{i}^{*}$ to the commutative semigroup $G_{i}^{*}$, where $g_{i}^{*} \tau=g_{i}^{*}$ for any $\tau \in G_{i}^{*}$ (the new elements $g_{i}^{*}$ are supposed to be different for different indices $i$ ). Then $G$ is a subsemigroup of the product of semigroups $G^{*}=\prod_{i \in I} G_{i}^{*}$ and the additive group of the semigroup ring $\mathbb{Z}\left[G^{*}\right]$ is a $\mathbb{Z}[G]$-module (the action of $G$ on $\mathbb{Z}\left[G^{*}\right]$ is given by the multiplication).

We introduce the group of circular numbers of $\mathbb{Q}\left(\zeta_{I}\right)$ and $\mathbb{Q}\left(\zeta_{I}\right)(q)$ as the following Galois modules

$$
\begin{aligned}
\mathcal{D} & =\left\langle 1-\zeta_{J} \mid \emptyset \neq J \subseteq I\right\rangle_{\mathbb{Z}[G]} \subset \mathbb{Q}\left(\zeta_{I}\right)^{\times} \\
\mathcal{D}_{(q)} & =\left\langle\eta_{J} \mid \emptyset \neq J \subseteq I \cup\{s+1\}\right\rangle_{\mathbb{Z}\left[G_{(q)}\right]} \subset \mathbb{Q}\left(\zeta_{I}\right)(q)^{\times},
\end{aligned}
$$

where $G_{(q)}=\operatorname{Gal}\left(\mathbb{Q}\left(\zeta_{I}\right)(q) / \mathbb{Q}\right)$ and

$$
\eta_{J}= \begin{cases}1-\zeta_{J} & \text { if } J \subseteq I, \\ \mathrm{~N}_{\mathbb{Q}\left(\zeta_{J}\right) / \mathbb{Q}\left(\zeta_{J-\{s+1\}}\right)(q)}\left(1-\zeta_{J}\right) & \text { otherwise. }\end{cases}
$$

Here we use the convention that $\mathbb{Q}\left(\zeta_{\emptyset}\right)$ means $\mathbb{Q}$. Admittedly it is not pretty but it does simplify notation.

Let $j$ be the complex conjugation. Consider the mappings $\pi: \mathcal{D} \rightarrow \mathcal{D}^{1+j}$ and $\pi_{(q)}: \mathcal{D}_{(q)} \rightarrow \mathcal{D}_{(q)}^{1+j}$ sending each $\varepsilon$ to $\varepsilon^{1+j}$. It is well known that the kernels of these mappings contain just the roots of unity, see [8, Lemma 4.1(i)], so ker $\pi=$ ker $\pi_{(q)}=\left\langle-\zeta_{I}\right\rangle$. Notice that the intersection of $\mathcal{D}^{1+j}$ and of the group of units of $\mathbb{Q}\left(\zeta_{I}\right)$ is equal to the Sinnott group of circular units of $\mathbb{Q}\left(\zeta_{I}\right)^{+}$, the maximal real subfield of $\mathbb{Q}\left(\zeta_{I}\right)$, see [6].

Theorem 5. There is a homomorphism of $\mathbb{Z}[G]$-modules $\Psi: \mathcal{D}^{1+j} \rightarrow \mathcal{D}_{(q)}^{1+j}$ satisfying $\Psi\left(\eta_{J}^{1+j}\right)=\eta_{J \cup\{s+1\}}^{1+j}$ for any nonempty $J \subseteq\{1, \ldots, s\}$.

Proof. We define a surjective $\mathbb{Z}[G]$-linear map $\vartheta: \mathbb{Z}\left[G^{*}\right] \rightarrow \mathcal{D}$ by

$$
\vartheta\left(\prod_{i \in I-J} g_{i}^{*}\right)= \begin{cases}1-\zeta_{J} & \text { if } J \neq \varnothing \\ 1 & \text { if } J=\varnothing\end{cases}
$$

for any $J \subseteq I$. Following [5] we define $\mathcal{I}_{3}$ as the $\mathbb{Z}[G]$-submodule of $\mathbb{Z}\left[G^{*}\right]$ generated by $(1-j) \mathbb{Z}\left[G^{*}\right]$ and by

$$
\left(s\left(G_{i}\right)-g_{i}^{*}\left(1-\lambda_{i}^{-1}\right)\right) \prod_{k \in V} g_{k}^{*}
$$


for all $i \in I$ and all $V \subsetneq I-\{i\}$. Using [5, Proposition 4.4 and Theorem 4.5], for each $Q \subseteq I,|Q|$ odd, $|Q|>1$, we have $\beta_{Q} \in \mathbb{Z}\left[G^{*}\right]$ such that $2 \beta_{Q} \in \mathcal{I}_{3}$ and that

$$
\operatorname{ker}(\pi \circ \vartheta)=\mathcal{I}_{3}+\left\langle\left\{\beta_{Q}|Q \subseteq I,| Q \mid \text { odd, }|Q|>1\right\} \cup\left\{g^{*}\right\}\right\rangle,
$$

where $g^{*}=\prod_{i \in I} g_{i}^{*}$. We define a $\mathbb{Z}[G]$-linear map $\vartheta_{(q)}: \mathbb{Z}\left[G^{*}\right] \rightarrow \mathcal{D}_{(q)}$ by

$$
\vartheta_{(q)}\left(\prod_{i \in I-J} g_{i}^{*}\right)= \begin{cases}\eta_{J \cup\{s+1\}} & \text { if } J \neq \varnothing, \\ 1 & \text { if } J=\varnothing,\end{cases}
$$

for any $J \subseteq I$. Since $\mathcal{I}_{3} \subseteq \operatorname{ker}\left(\pi_{(q)} \circ \vartheta_{(q)}\right)$ and $\mathcal{D}_{(q)}^{1+j}$ has no $\mathbb{Z}$-torsion, we get $\operatorname{ker}(\pi \circ \vartheta) \subseteq \operatorname{ker}\left(\pi_{(q)} \circ \vartheta_{(q)}\right)$. Since $\pi \circ \vartheta$ is surjective, this inclusion implies there is a $\mathbb{Z}[G]$-linear map $\Psi: \mathcal{D}^{1+j} \rightarrow \mathcal{D}_{(q)}^{1+j}$ satisfying $\Psi\left(\eta_{J}^{1+j}\right)=\eta_{J \cup\{s+1\}}^{1+j}$ for any $\emptyset \neq J \subseteq I$.

Theorem 6. Let $\operatorname{Frob}(q)$ be the Frobenius automorphism of $q$ on $\mathbb{Q}\left(\zeta_{I}\right)$. For any $\varepsilon \in \mathcal{D}^{1+j}$ we have

$$
\begin{aligned}
\varepsilon^{(q-1) / M} & \equiv \Psi\left(\varepsilon^{\operatorname{Frob}(q)}\right) \text { modulo each prime ideal of } \mathbb{Q}\left(\zeta_{I}\right)(q) \text { above } q, \\
\mathrm{~N}_{\bar{K}(q) / \bar{K}}(\Psi(\varepsilon)) & =\varepsilon^{1-\operatorname{Frob}(q)^{-1}} .
\end{aligned}
$$

Proof. This can be proven using (1) just as Theorem 2.

Corollary 7. For any $\varepsilon \in \mathcal{D}^{1+j} \cap K$ we have $\Psi(\varepsilon) \in K(q)$,

$$
\varepsilon^{(q-1) / M} \equiv \Psi(\varepsilon) \text { modulo each prime ideal of } \bar{K}(q) \text { above } q,
$$

and $\mathrm{N}_{\bar{K}(q) / \bar{K}}(\Psi(\varepsilon))=1$.

Proof. See the proof of Corollary 3.

Proposition 8. Every $\varepsilon \in K$ which can be written as $u^{1+j}$ with some $u \in \mathcal{D}$ is $M$-semispecial for every $\ell$-power $M$, and in particular semispecial (with respect to any odd prime $\ell$ ). This applies in particular if $\varepsilon$ is the square of a real Washington unit in $K$.

Proof. See the proof of Proposition 4.

\section{Semispecial elements and annihilation}

We begin this section by stating a close analog of Theorem 12 in [1], which will be an important tool in ongoing work of the authors (for more details please see below). There have been several previous occasions where we needed to adapt certain results of this type to fit a slightly larger framework, and so far we have painstakingly explained what needs to be done. However, the case at hand is so straightforward that a few remarks should suffice to explain why all main steps of the proof given in loc.cit. remain valid in our setting. It is remarkable, and perhaps even a little bit embarrassing, that almost none of the conditions that we imposed on $K$ in loc.cit. are actually needed. We continue to assume that $\ell$ is a fixed odd prime, unless otherwise stated. 
Theorem 9. Let $K$ be any abelian field in which $\ell$ is unramified, and let $G=$ $\mathrm{Gal}(K / \mathbb{Q})$. Fix a large $\ell$-power $M$, and assume that $\varepsilon \in K^{\times}$is $M$-semispecial. Moreover suppose that $V \subset K^{\times} /\left(K^{\times}\right)^{M}$ is a finitely generated $\mathbb{Z}[G]$-submodule containing $\varepsilon$. Let $\alpha: V \rightarrow \mathbb{Z} / M[G]$ be any $\mathbb{Z}[G]$-linear map whose restriction to $V \cap \mathbb{Q}$ is zero. Then $\alpha(\varepsilon)$ annihilates $C l(K) /(M \cdot C l(K))$.

Remark. The abuse of notation $V \cap \mathbb{Q}$ seems to go back to Rubin; it stands for the intersection of $V$ and $\mathbb{Q}^{\times}\left(K^{\times}\right)^{M} /\left(K^{\times}\right)^{M}$ inside $K^{\times} /\left(K^{\times}\right)^{M}$. When we apply the theorem in our companion paper [3], $V$ will actually be the image of the unit group $\mathcal{O}_{K}^{\times}$, so it is possible to identify $V$ with $\mathcal{O}_{K}^{\times}\left(K^{\times}\right)^{M} /\left(K^{\times}\right)^{M}$. We would like to mention an interesting and somewhat surprising point: Even though $\mathcal{O}_{K}^{\times}$has no $G$-fixed elements (except \pm 1 which do not matter modulo $M$ ), the quotient $\mathcal{O}_{K}^{\times}\left(K^{\times}\right)^{M} /\left(K^{\times}\right)^{M}$ always has nontrivial $G$-fixed elements (this is true for any $\ell$ group acting on any nonzero finite $\mathbb{Z}_{\ell}$-module), and one may show that this implies: $V \cap \mathbb{Q}$ is nontrivial as well. So the condition that $\alpha$ has to be trivial on $V \cap \mathbb{Q}$ cannot be ignored even if $V$ is made up of units.

\section{Comments on the proof:}

All references are to [1]. Let us simply scan the proof of Theorem 12, as written up there from below middle of page 193 until the end of text; the main issue is to make sure that the auxiliary results still hold. Theorem 17 remains literally true. The technical core in the argument are the disjointness statements gathered in Lemma 18. In the proof of part (a), the main point is that the Galois group of $K\left(\zeta_{M^{2}}\right) / K$ is as large as it can be, that is, isomorphic to $\left(\mathbb{Z} / M^{2}\right)^{\times}$. And this is insured by just assuming that the odd prime $\ell$ (which is denoted $p$ in loc.cit.) does not ramify in $K$. The same goes for part (b), and the argument for (c) has nothing to do with $G$ being cyclic or not. Obviously, the definition of $e_{0}$ on line 8 of p.195 has to be rewritten; the proper way is of course $e_{0}\left(\sum_{\sigma \in G} a_{\sigma} \cdot \sigma\right):=\zeta_{M}^{a_{1}}$, where 1 is (abusive) notation for the neutral element of $G$. The last ingredient is Theorem 19, which comes from a paper of Rubin, and there $K$ (written $F$ in that paper) was supposed to be abelian over the base field (written $K$ in that paper) and this base field is just $\mathbb{Q}$ in our case. This concludes our review of the proof given in loc.cit.

Let us make one very technical remark, for the benefit of those readers which are familiar with [1] or actually go through our comparison arguments. We no longer need to use the auxiliary quantity $M^{\prime}=M / \ell^{s-1}$. This makes the proof simpler; the semispeciality condition, as formulated and proved in the present paper, now gives a congruence modulo $M$, not $M^{\prime}$, and the transition from "modulo $M$ " to "modulo $M^{\prime \prime}$ " that was required on page 197 of loc.cit. disappears.

Before we continue, let us mention in passing that it is possible to state and prove an analog of the preceding theorem for $\ell=2$. This was done by Herman [4]. To make this work, a few technical changes are necessary; let us only mention the most important one. In the definition of "semispecial", the congruence $q \equiv 1+M$ $\left(\bmod M^{2}\right)$ has to be replaced by $q \equiv 1+2 M\left(\bmod M^{2}\right)$. Observe that now $M$ is a 2-power.

Let us now describe to what purpose we will use the previous result in ongoing work [3]. There we study a certain class of real abelian fields, which we now define. Let $\ell$ be a fixed odd prime, $t>1$ an integer, and $K_{1}, \ldots, K_{t}$ a list of $t$ abelian fields 
of degree $\ell$ over $\mathbb{Q}$ (in particular $\operatorname{Gal}\left(K_{i} / \mathbb{Q}\right)$ is cyclic for all $1 \leq i \leq t$ ), and denote by $P_{i}$ the set of rational primes that ramify in $K_{i}$. We moreover assume that the sets $P_{i}(1 \leq i \leq t)$ are mutally disjoint and do not contain $\ell$. Let $K=K_{1} \cdots K_{t}$ be the compositum of the $K_{i}$ and let $G=\operatorname{Gal}(K / \mathbb{Q})$. Recall that a Washington unit of $K$ is a unit of $K$ which is also a cyclotomic number in the conductor-level cyclotomic field.

We now impose an extra condition. Two abelian fields $K^{\prime}$ and $K^{\prime \prime}$ are called arithmetically orthogonal (just orthogonal for short) if every prime that ramifies in $K^{\prime}$ is totally split in $K^{\prime \prime}$ and vice versa. We demand that the fields $K_{1}, \ldots, K_{t}$ are pairwise orthogonal and that at least one of the sets $P_{i}$ has more than one element. This hypothesis allows us to find "unexpected" Washington units, and leads to sharper annihilation results, in the style of Thaine and Rubin, than those afforded by Sinnott units. It has been known that the latter are semispecial, but the more semispecial units we have, the better the annihilation results will turn out.

A little more precisely: Under the above orthogonality assumption, we show that there are specific Washington units $\varepsilon_{T} \in K$, one for each nonempty subset $T$ of $\{1, \ldots, t\}$, such that the group $\bar{C}$ obtained by adjoining all conjugates of all $\varepsilon_{T}$ to $C_{K}$ (the group of Sinnott units of $K$ ) is much larger than $C_{K}$ (of course this is quantified precisely). The existence proof for these new Washington units is rather long and technical. (By the way, this is one of the main reasons for distributing our material over two papers.) To give the reader an idea of the strength of this result, we mention one consequence. If $s$ denotes the cardinality of the union $P_{1} \cup \ldots \cup P_{t}$, then we obtain that the class number $h_{K}$ is divisible by $\ell^{(s-t) \ell^{t-1}}$. We also prove a result in the spirit of Thaine and Rubin, stating that up to an explicit technical factor the annihilator of $\mathcal{O}_{K}^{\times} / \bar{C}$ annihilates the $\ell$-part of the class group of $K$. Moreover we will give numerical examples for these phenomena in [3], but for this we have to restrict ourselves to $t=2$ and $\ell=3$ since otherwise the degree of $K$ would become too large.

Acknowledgements The second author was supported under Project 18-11473S of the Czech Science Foundation.

Open Access This article is licensed under a Creative Commons Attribution 4.0 International License, which permits use, sharing, adaptation, distribution and reproduction in any medium or format, as long as you give appropriate credit to the original author(s) and the source, provide a link to the Creative Commons licence, and indicate if changes were made. The images or other third party material in this article are included in the article's Creative Commons licence, unless indicated otherwise in a credit line to the material. If material is not included in the article's Creative Commons licence and your intended use is not permitted by statutory regulation or exceeds the permitted use, you will need to obtain permission directly from the copyright holder. To view a copy of this licence, visit http://creativecommons.org/ licenses/by/4.0/.

Funding Open access funding provided by Projekt DEAL. 


\section{References}

[1] Greither, C., Kučera, R.: Annihilators for the class group of a cyclic field of prime power degree. Acta Arith. 112, 177-198 (2004)

[2] Greither, C., Kučera, R.: Linear forms on Sinnott's module. J. Number Theory 141, 324-342 (2014)

[3] Greither, C., Kučera, R.: On the compositum of orthogonal cyclic fields of the same odd prime degree. Can. J. Math. (to appear)

[4] Herman, J.: Annihilators of the class group of a compositum of quadratic fields. Arch. Math. (Brno) 49, 209-222 (2013)

[5] Kučera, R.: Circular units and Stickelberger ideal of a cyclotomic field revisited. Acta Arith. 174, 217-238 (2016)

[6] Lettl, G.: A note on Thaine's circular units. J. Number Theory 35, 224-226 (1990)

[7] Rubin, K.: Global units and ideal class groups. Invent. Math. 89, 511-526 (1987)

[8] Sinnott, W.: On the Stickelberger ideal and the circular units of an abelian field. Invent. Math. 62, 181-234 (1980)

Publisher's Note Springer Nature remains neutral with regard to jurisdictional claims in published maps and institutional affiliations. 